\title{
RADIUS OF UNIVALENCE AND STARLIKENESS OF A CLASS OF ANALYTIC FUNCTIONS
}

\author{
R. S. GUPTA
}

(Received 10 April 1970)

Communicated by E. Strzelecki

Let $\mathscr{P}$ denote the family of functions

$$
P(z)=1+2 a_{1} z+a_{2} z^{2}+\cdots
$$

regular in $E\{z:|z|<1\}$ and with positive real part there. We propose to study, in this article, the subclass $\mathscr{P}_{2 a_{1}}$ of $\mathscr{P}$ whose functions $P(z)$ have pre-assigned second coefficient $2 a_{1}$. In what follows we may assume, without loss in generality, that $a_{1}$ is real and non-negative. This assumption will be made throughout. As is well known [2], $0 \leqq a_{1} \leqq 1$. In Theorem 1 we derive a generalization of Zmorovic's theorem 1, [3]. The result so obtained is then utilized in Theorem 2 to determine the radius of univalence and starlikeness of the class of functions

$$
\Gamma(z)=P(z)-1=2 a_{1} z+a_{2} z^{2}+\cdots
$$

where $P(z) \in \mathscr{P}_{2 a_{1}}$.

TheOREM 1. Let $P \in \mathscr{P}_{2 a_{1}}$. Then we have on $|z|=r$

$$
\left|z P^{\prime}-\frac{P^{2}-1}{2}\right| \leqq \frac{\rho^{2}-\rho_{0}^{2}}{2}
$$

where

$$
\rho=\frac{2 r}{1-r^{2}}, \quad a=\frac{1+r^{2}}{1-r^{2}}, \quad|P-a|=\rho_{0} \leqq \rho
$$

This estimate is sharp.

Proof. We shall first prove (1.1) for the general class $\mathscr{P}$. The proof of the theorem will then be completed by showing the existence of a function belonging to $\mathscr{P}_{2 a_{1}}$ for which equality holds in (1.1). We first observe that if $\phi(z)$ is regular and bounded in $E$, (by the term 'bounded' we shall always mean 'bounded by one') with $\phi(0)=0$, then the function 


$$
\psi(z)=\frac{\phi(z)}{z}
$$

is likewise bounded in $E$. Differentiating (1.2) we obtain

$$
\psi^{\prime}(z)=\frac{\phi^{\prime}(z)}{z}-\frac{\phi(z)}{z^{2}}
$$

Therefore, [2],

$$
\left|\frac{\phi^{\prime}(z)}{z}-\frac{\phi(z)}{z^{2}}\right|=\left|\psi^{\prime}(z)\right| \leqq \frac{1-|\psi(z)|^{2}}{1-r^{2}}
$$

Substituting the value of $\psi(z)$ from (1.2) and simplifying, (1.4) yields

$$
\left|z \phi^{\prime}(z)-\phi(z)\right| \leqq \frac{r^{2}-|\phi(z)|^{2}}{1-r^{2}}
$$

Now

$$
\phi(z)=\frac{P(z)-1}{P(z)+1}
$$

from which we obtain after differentiation

$$
z \phi^{\prime}(z)=\frac{2 z P^{\prime}(z)}{(P(z)+1)^{2}}
$$

Substituting (1.6) and (1.7) in (1.5) we get

$$
\left|z P^{\prime}-\frac{P^{2}-1}{2}\right| \leqq \frac{r^{2}(|P+1|)^{2}-|P-1|^{2}}{2\left(1-r^{2}\right)}=\frac{\left(\frac{2 r}{1-r^{2}}\right)^{2}-\left|P-\frac{1+r^{2}}{1-r^{2}}\right|^{2}}{2}
$$

This gives (1.1) for $P \in \mathscr{P}$. For the class $\mathscr{P}_{2 a_{1}}$ it is readily verified that the function

$$
P_{0}(z)=\frac{1+2 a_{1} z+z^{2}}{1-z^{2}}
$$

belonging to $\mathscr{P}_{2 a_{1}}$ yields equality in (1.1). Thus the estimate (1.1) holds for $P \in \mathscr{P}_{2 a_{1}}$ and the proof of the theorem is complete.

It may be remarked that $Z$ morovic [3] proved the inequality (1.1) under the condition that the function $P(z) \in \mathscr{P}$ has the form

$$
P(z)=\lambda_{1} \frac{1+z_{1}^{m}}{1-z_{1}^{m}} \cdot+\lambda_{2} \frac{1+z_{2}^{m}}{1-z_{2}^{m}},
$$

where $z_{1}$ and $z_{2}$ are arbitrary points on $|z|=r, m$ is a positive integer, $\lambda_{1} \geqq 0, \lambda_{2} \geqq 0, \lambda_{1}+\lambda_{2}=1$. We have proved (1.1) without this assumption.

THEOREM 2. Let $P \in \mathscr{P}_{2 a_{1}}$. Then the radius of univalence and starlikeness, $r_{0}$ of the class of functions 


$$
\Gamma(z)=P(z)-1=2 a_{1} z+a_{2} z^{2}+\cdots
$$

is given by

$$
r_{0}=\frac{a_{1}}{1+\sqrt{1-a_{1}^{2}}}
$$

In order to prove the theorem we need the following

LeMma 1. Let $P \in \mathscr{P}_{2 a_{1}}$. Then on $|z|=r<a_{1}$, we have

$$
|P-1-x| \geqq y
$$

where $x=\frac{2 r^{2}\left(a_{1}-r\right)^{2}}{\left(1-r^{2}\right)\left(1-2 a_{1} r+r^{2}\right)}$ and $y=\frac{2 r\left(a_{1}-r\right)\left(1-a_{1} r\right)}{\left(1-r^{2}\right)\left(1-2 a_{1} r+r^{2}\right)}$

This result is sharp in the sense that $P-1$ take the value

$$
A=\frac{-2 r\left(a_{1}-r\right)}{1-r^{2}} \text { and } B=\frac{2 r\left(a_{1}-r\right)}{1-2 a_{1} r+r^{2}}
$$

which are the extremities of the diameter of the circle $A L B$ whose centre is at $x$ and radius is $y$.

Proof. For $P \in \mathscr{P}_{2 a_{1}}$, we may write

$$
\frac{P-1}{P+1}=\phi(z)
$$

where $\phi(z)$ is regular and bounded in $E, \phi(0)=0, \phi^{\prime}(0)=a_{1}$, from which it follows that, [2]

$$
\left|\frac{P-1}{P+1}\right|=|\phi(z)| \geqq \frac{r\left(a_{1}-r\right)}{1-a_{1} r}
$$

and this yields

$$
\left|P-\frac{1-2 a_{1} r+2 a_{1}^{2} r^{2}-2 a_{1} r^{3}+r^{4}}{\left(1-r^{2}\right)\left(1-2 a_{1} r+r^{2}\right)}\right| \geqq y
$$

which is equivalent to (2.3). The values $A$ and $B$ are respectively taken by the functions

$$
\frac{1+2 a_{1} z+z^{2}}{1-z^{2}} \quad \text { at } z=-r
$$

and

$$
\frac{1-z^{2}}{1-2 a_{1} z+z^{2}} \quad \text { at } z=+r
$$

This completes the proof of the lemma. 
Lemma 2. Let $P \in \mathscr{P}_{2 a_{1}}$. Then on $|z|=r, 0<r<1$ we have

$$
|P-1-X| \leqq Y
$$

where

$$
X=\frac{2 r^{2}\left(a_{1}+r\right)^{2}}{\left(1+2 a_{1} r+r^{2}\right)\left(1-r^{2}\right)} \text { and } Y=\frac{2 r\left(1+a_{1} r\right)\left(a_{1}+r\right)}{\left(1+2 a_{1} r+r^{2}\right)\left(1-r^{2}\right)}
$$

This result is sharp in the sense that $P-1$ take the values

$$
A^{\prime}=\frac{-2 r\left(a_{1}+r\right)}{1+2 a_{1} r+r^{2}} \quad \text { and } B^{\prime}=\frac{2 r\left(a_{1}+r\right)}{1-r^{2}}
$$

which are the extremities of the diameter of the circle $A^{\prime} L^{\prime} B^{\prime}$ whose centre is at $X$ and radius is $Y$.

Proof. The proof is similar to that of Lemma 1 and follows from the inequality, [2],

$$
\left|\frac{P-1}{P+1}\right|=|\phi(z)| \leqq \frac{r\left(a_{1}+r\right)}{1+a_{1} r}
$$

where $\phi(z)$ is regular and bounded in $E, \phi(0)=0, \phi^{\prime}(0)=a_{1}$. The functions of Lemma 1 are extremal in this case as well.

Proof of Theorem 2. The function $\Gamma(z)=P(z)-1$ is regular in $E$ and from Lemma 1 we see that

$$
\left|\frac{P(z)-1}{z}\right| \geqq \frac{2\left(a_{1}-r\right)}{1-r^{2}}
$$

so that $\Gamma(z)$ has no zeros in $|z|<a_{1}$ except a simple zero at the origin. A necessary and sufficient condition that $\Gamma(z)$ be starlike in $|z|<r_{0}$ is that

$$
\operatorname{Re} \frac{z \Gamma^{\prime}(z)}{\Gamma(z)}=\operatorname{Re} \frac{z P^{\prime}(z)}{P(z)-1}>0
$$

in $|z|<r_{0}$. Since $\operatorname{Re} \frac{z P^{\prime}(z)}{P(z)-1}$ is harmonic in $|z|<a_{1}$, it is sufficient to obtain the radius of the largest circle on which this is non-negative.

Making use of (1.1) we get

$$
\operatorname{Re} \frac{z P^{\prime}}{P-1} \geqq \operatorname{Re} \frac{P+1}{2}-\frac{\rho^{2}-\rho_{0}^{2}}{2|P-1|}
$$

We now have the following extremal problem: Given $|z|=r$, to find the minimum of the right side of (2.9) as $P$ runs over the class $\mathscr{P}_{2 a_{1}}$. From Lemma 1 and 2 we see that we need to find this minimum for $P-1$ lying in the region enclosed by the circles $A^{\prime} L^{\prime} B^{\prime}$ and $A L B$. 
Putting $P-a=\xi+i \eta$ and denoting the right side of $(2.9)$ by $\psi_{\rho}(\xi, \eta)$ we obtain

$$
\psi_{\rho}(\xi, \eta)=\frac{1}{2}\left[a+\xi+1-\frac{\rho^{2}-\left(\xi^{2}+\eta^{2}\right)}{R}\right]
$$

where $R=|P-1|$.

We now divide the range of $\xi=\operatorname{Re}(P-a)$ into two parts:

$$
\xi \leqq \frac{-2 a_{1} r}{1-r^{2}} \text { and } \xi \geqq \frac{2 r\left(a_{1}-2 r+a_{1} r^{2}\right)}{\left(1-2 a_{1} r+r^{2}\right)\left(1-r^{2}\right)}
$$

and

$$
-\frac{2 a_{1} r}{1-r^{2}} \leqq \xi \leqq \frac{2 r\left(a_{1}-2 r+a_{1} r^{2}\right)}{\left(1-2 a_{1} r+r^{2}\right)\left(1-r^{2}\right)}
$$

We shall show that in case (A) the minimum of $\psi_{\rho}(\xi, \eta)$ inside the circle $\xi^{2}+\eta^{2}$ $=\rho_{0}^{2} \leqq \rho^{2}$ is attained on the diameter $\eta=0$. We differentiate $(2.10)$ with respect to $\eta$ and obtain

$$
\frac{\partial \psi}{\partial \eta}=\frac{1}{2}\left[\frac{\rho^{2}-\left(\xi^{2}+\eta^{2}\right)}{R^{2}} \cdot \frac{\eta}{R}+\frac{2 \eta}{R}\right]=\frac{\eta}{2 R^{3}}\left[\rho^{2}-\left(\xi^{2}+\eta^{2}\right)+2 R^{2}\right]
$$

The expression within the brackets is positive. Hence for each fixed $\xi$, the minimum is attained at $\eta=0$. Therefore, inside the circle $\xi^{2}+\eta^{2}=\rho_{0}^{2}$ (subject to (A)) the minimum occurs on the diameter $\eta=0$. of

Putting $\eta=0$ in (2.10) we have the following problem: To find the minimum

$$
l(\xi)=\frac{1}{2}\left[a+\xi+1-\frac{\rho^{2}-\xi^{2}}{|a+\xi-1|}\right]
$$

If $\xi \leqq-2 a_{1} r /\left(1-r^{2}\right)$, then $a+\xi-1$ is negative and so

$$
l(\xi)=\frac{1}{2}\left[a+\xi+1+\frac{\rho^{2}-\xi^{2}}{a+\xi-1}\right]=\frac{2 r^{2}}{\left(1-r^{2}\right)(a+\xi-1)}+\frac{1+r^{2}}{1-r^{2}}
$$

from which we see that the minimum of $l(\xi)\left(=l_{1}(\xi)\right) l$ is given by the smallest numerical value of $a+\xi-1$. Substituting $\xi=-2 a_{1} r /\left(1-r^{2}\right)$ in (2.13) we obtain

If

$$
l(\xi) \geqq l_{1}(\xi)=\frac{a_{1}-2 r+a_{1} r^{2}}{\left(a_{1}-r\right)\left(1-r^{2}\right)}
$$

$$
\xi \geqq \frac{2 r\left(a_{1}-2 r+a_{1} r^{2}\right)}{\left(1-2 a_{1} r+r^{2}\right)\left(1-r^{2}\right)},
$$

then $a+\xi-1$ is positive. In this case 


$$
\begin{aligned}
l(\xi) & =\frac{1}{2}\left[a+\xi+1-\frac{\rho^{2}-\xi^{2}}{a+\xi-1}\right] \\
& =a+\xi-1-\frac{2 r^{2}}{\left(1-r^{2}\right)(a+\xi-1)}+\frac{1-3 r^{2}}{1-r^{2}}
\end{aligned}
$$

Since $a+\xi-1>0$, the minimum of $l(\xi)\left(=l_{2}(\xi)\right)$ occurs for the smallest numerical value of $a+\xi-1$. Putting

$$
\xi=\frac{2 r\left(a_{1}-2 r+a_{1} r^{2}\right)}{\left(1-2 a_{1} r+r^{2}\right)\left(1-r^{2}\right)}
$$

in (2.15) we obtain

$$
l(\xi) \geqq l_{2}(\xi)=\frac{2 r\left(a_{1}-r\right)}{1-2 a_{1} r+r^{2}}-\frac{2 r^{2}\left(1-2 a_{1} r+r^{2}\right)}{\left(1-r^{2}\right) \cdot 2 r\left(a_{1}-r\right)}+\frac{1-3 r^{2}}{1-r^{2}}
$$

From (2.14) and (2.16) we see that

$$
l_{2}(\xi) \geqq l_{1}(\xi)
$$

if

$$
a_{1}-2 r+a_{1} r^{2} \geqq 0, \text { that is, if } r \leqq a_{1} /\left(1+\sqrt{1-a_{1}^{2}}\right)
$$

In case (B) let us assume that every value on the circumference of the circle $A L B$ is taken by some $P-1, P \in \mathscr{P}_{2 a_{1}}$, for some $z,|z|=r<a_{1}$. We see then from (2.11) that for each fixed $\xi$, the minimum of the right side of (2.9) occurs for points on the circumference of the circle $A L B$. Therefore, for the admissible range of $\xi$, the minimum occurs on the circumference of the above circle. Also, from (2.3) we see that any point on the circumference of the circle can be written as

$$
P-1=x+y e^{i \theta} \quad 0 \leqq \theta<2 \pi
$$

so that our problem reduces to minimizing the expression:

$$
\begin{aligned}
\psi & =1+\frac{1}{2}\left[\operatorname{Re}(P-1)-\frac{\rho^{2}-|P-a|^{2}}{|P-1|}\right] \\
& =1+\frac{1}{2}\left[x+y \cos \theta-\left(\frac{4 r^{2}}{\left(1-r^{2}\right) \sqrt{2 x}}-\sqrt{2 x}\right)(1+x+y \cos \theta)^{\frac{1}{2}}\right]
\end{aligned}
$$

where we have made use of the fact that

$$
x^{2}-y^{2}+2 x \equiv 0
$$

Differentiating (2.18) with respect to $\theta$ we obtain

(2.20) $\frac{\partial \psi}{\partial \theta_{i}}=-\frac{1}{2} y \sin \theta\left[1-\frac{1}{2}\left(\frac{4 r^{2}}{\left(1-r^{2}\right) \sqrt{2 x}}-\sqrt{2 x}\right)(1+x+y \cos \theta)^{-\frac{1}{2}}\right]$ 
We propose to show that the expression within the brackets retains a positive sign at least when $|z|=r<a_{1} /\left(1+\sqrt{1-a_{1}^{2}}\right)$. It will then follow that the minimum of $\psi$ can occur only when $\theta=0$ or $\theta=\pi$, that is, at $A$ or $B$. In other words, the minimum of $\psi$ in case (A) and case (B) is the same if $|z|=r<a_{1} /\left(1+\sqrt{1-a^{2}}\right)$.

To show that the expression within the brackets retains a positive sign, let us put

$$
\Phi=1-\frac{1}{2}\left(\frac{4 r^{2}}{\left(1-r^{2}\right) \sqrt{2 x}}-\sqrt{2 x}\right) \cdot(1+x+y \cos \theta)^{-\frac{1}{2}}
$$

then

$$
\begin{aligned}
\frac{\partial \Phi}{\partial \theta} & =-\frac{1}{4} y \sin \theta\left(\frac{4 r^{2}}{\left(1-r^{2}\right) \sqrt{2 x}}-\sqrt{2 x}\right) \cdot(1+x+y \cos \theta)^{-\frac{3}{2}} \\
& =x y \sin \theta\left(x-\frac{2 r^{2}}{1-r^{2}}\right) \cdot\left(x^{2}+y^{2}+2 x y \cos \theta\right)^{-3}
\end{aligned}
$$

Since

$$
x-\frac{2 r^{2}}{1-r^{2}}=-\frac{2 r^{2}}{\left(1-r^{2}\right)} \frac{\left(1-a_{1}^{2}\right)}{\left(1-2 a_{1} r+r^{2}\right)}, x^{2}+y^{2}+2 x y \cos \theta>0,
$$

the extrema of $\Phi$ occurs for $\theta=0, \pi$ (if $a_{1}=1, \Phi \equiv 1$ ). For $\theta=0$,

For $\theta=\pi$

$$
\Phi=\Phi_{0}=\frac{a_{1}-2 r-a_{1} r^{2}+a_{1}^{2} r+r^{3}}{\left(a_{1}-r\right)\left(1-r^{2}\right)}
$$

$$
\Phi=\Phi_{\pi}=\frac{a_{1}-3 a_{1}^{2} r+3 a_{1} r^{2}-r^{3}}{\left(a_{1}-r\right)\left(1-2 a_{1} r+r^{2}\right)}
$$

and we will show that when $r<a_{1} /\left(1+\sqrt{ } 1-a_{1}^{2}\right)$ both $\Phi_{0}$ and $\Phi_{n}$ are positive. The numerator of

$$
\begin{aligned}
\Phi_{0} & =a_{1}-2 r+a_{1} r^{2}-2 a_{1} r^{2}+a_{1}^{2} r+r^{3} \\
& \geqq a_{1}-2 r+a_{1} r^{2}-2 a_{1} r^{2}+a_{1}^{2} r+a_{1}^{2} r^{3} \\
& =\left(a_{1}-2 r+a_{1} r^{2}\right)\left(1-a_{1} r\right)>0 \quad \text { if } r<a_{1} /\left(1+\sqrt{1-a_{1}^{2}}\right)
\end{aligned}
$$

If $a_{1}=1$, it is easy to see that $\Phi_{\pi}=1$. Otherwise the numerator of $\Phi_{\pi}$ is a monotonic decreasing function of $r$. Putting $r=a_{1}$, the numerator becomes $a_{1}-a_{1}^{3}>0$. Therefore if $r<a_{1} /\left(1+\sqrt{1-a_{1}^{2}}\right)<a_{1}, \phi_{\pi}$ is also positive.

Finally, if $P-1$ omits a larger set of values than the interior of the circle $A L B$, this omitted set of values will include the interior of the above circle but not the points $A$ and $B$ and so the minimum $/$ max will again occur at $A$ or $B$.

Summing up, we have proved that for $|z|=r<a_{1} /\left(1+\sqrt{1-a^{1}}\right)$

$$
\operatorname{Re}_{P \in \mathscr{F}_{2},} \frac{z P^{\prime}}{P-1} \geqq \frac{a_{1}-2 r+a_{1} r^{2}}{\left(a_{1}-r\right)\left(1-r^{2}\right)}
$$


Also the right side of this inequality is non-negative for

$$
|z| \leqq r_{0}=a_{1} /\left(1+\sqrt{1-a_{1}^{2}}\right) \text {. }
$$

Therefore $\Gamma(z)$ is starlike in $|z|<r_{0}$. That $\Gamma(z)$ may not be starlike in a larger circle may be shown by considering the function

$$
P_{0}(z)=\frac{1+2 a_{1} z+z^{2}}{1-z^{2}} \in \mathscr{P}_{2 a_{1}}
$$

for which $\operatorname{Re} z P^{\prime} /(P-1)$ vanishes on $\mid z_{\mid}=r_{0}$. Thus the estimate (2.2) for the radius of starlikeness is correct. Since the derivative of $P_{0}(z)$ vanishes for $|z|=r_{0}=a_{1} /\left(1+\sqrt{1-a_{1}^{2}}\right)$, we see that $r_{0}$ is also the radius of univalence of the class $\Gamma(z)=P(z)-1, P \in \mathscr{P}_{2 a_{1}}$. This completes the proof of the theorem.

It may be pointed out that the radius of univalence of $\Gamma(z)$ follows immediately from a result of Landau [1] who showed that a function $\phi(z)=a_{1} z+\cdots$ which is regular and bounded in $E$ is univalent in the disc

$$
|z|<\frac{\left|a_{1}\right|}{1+\sqrt{1-\left|a_{1}\right|^{2}}}
$$

Since we may write $\Gamma(z)=P(z)-1=2 \phi /(1-\phi)$ where $P \in \mathscr{P}_{2 a_{1}}, \phi$ is regular and bounded in $E$ and $\phi(0)=0, \phi^{\prime}(0)=a_{1}$, the univalence of $\Gamma(z)$ in the $\operatorname{disc}(2.22)$ follows from the univalence of $\phi$ in the same disc. Of course, insofar as starlikeness is concerned the situation for $\Gamma(z)$ and $\phi(z)$ would be quite different because of the intervention of the linear transformation.

\section{Acknowledgements}

In the end the author wishes to express his gratitude to his guide Dr. V. Singh for his help in the preparation of this paper, particularly for the proof of Theorem 1 which is due to him. His thanks are also due to the referee for his suggestions leading to improvement of the paper.

\section{References}

[1] E. Landau, 'Der Picard-Schottkysche Satz un die Blochsche Konstante', Sitzungsb. Akad. d. Wiss. Berlin, Phys. Math. Klasse (1926), 467-474.

[2] Z. Nehari, Conformal Mapping (McGraw-Hill, 1952).

[3] V. A. Zmorovic, 'On the radius of convexity of starlike functions of order a regular in $|z|<1$ and in $0<|z|<1$ ' (Russian), Mat. Sbornik (N. S.) 68 (110) (1965), 518-526.

Department of Mathematics

Punjabi University

Patiala, India 